\title{
Fuzzy-Art Neural Networks for Predicting Chi-Chi Earthquake Induced Liquefaction in Yuan-Lin Area
}

\section{S. G. Chern}

Professor, Department of Harbor and River Engineering, National Taiwan Ocean University, Keelung, Taiwan, R.O.C.

R. F. Hu

Graduate Student, Department of Harbor and River Engineering, National Taiwan Ocean University, Keelung, Taiwan, R.O.C.

\section{Y. J. Chang}

Graduate Student, Department of Harbor and River Engineering, National Taiwan Ocean University, Keelung, Taiwan, R.O.C.

\section{F. Tsai}

Graduate Student, Department of Harbor and River Engineering, National Taiwan Ocean University, Keelung, Taiwan, R.O.C.

Follow this and additional works at: https://jmstt.ntou.edu.tw/journal

Part of the Civil and Environmental Engineering Commons

\section{Recommended Citation}

Chern, S. G.; Hu, R. F.; Chang, Y. J.; and Tsai, I. F. (2002) "Fuzzy-Art Neural Networks for Predicting Chi-Chi Earthquake Induced Liquefaction in Yuan-Lin Area," Journal of Marine Science and Technology. Vol. 10: Iss. 1, Article 4.

DOI: 10.51400/2709-6998.2297

Available at: https://jmstt.ntou.edu.tw/journal/vol10/iss1/4

This Research Article is brought to you for free and open access by Journal of Marine Science and Technology. It has been accepted for inclusion in Journal of Marine Science and Technology by an authorized editor of Journal of Marine Science and Technology. 


\title{
FUZZ-ART NEURAL NETWORKS FOR PREDICTING CHI-CHI EARTHQUAKE INDUCED LIQUEFACTION IN YUAN-LIN AREA
}

\author{
S. G. Chern*, R. F. Hu**, Y. J. Chang**, and I. F. Tsai**
}

Key words: Fuzz-ART, ART, fuzzy rule, geotechnical engineering.

\begin{abstract}
In this study, a fuzzy adaptive network "Fuzz-ART", based on adaptive resonance theory (ART) combined with fuzzy set theory is developed to evaluate liquefaction potentials induced by Chi-Chi earthquake in Yuan-Lin area. The proposed system combines the backpropagation algorithm for parameter learning and the fuzzy ART algorithm for structure learning. With the help of case studies, it is shown that the "Fuzz-ART" network is able to predict liquefaction potentials much more satisfactorily than conventional artificial neural network methods. If more data are collected, it may well evaluate liquefaction potentials induced by $\mathrm{Chi}$-Chi earthquake.
\end{abstract}

\section{INTRODUCTION}

There are hundreds of recent cases of ground failure and damage to structures due to liquefaction during earthquake in Taiwan, Japan, Yugaslavia, Chile, China, Central America and the United States. During the 1964 and 1995 earthquakes in Niigata and Kobe, Japan, many structures settled several feet and suffered up to 80 degrees of tilting (Oshaki, 1970; Yomamuro et al., 1997). The same year of Niigata earthquake, in Valdez, Alaska, extensive flow slides washed entire sections of the waterfront into the sea. Recently in 1999, liquefaction caused a considerable amount of damage in the central part of Taiwan during Chi-Chi earthquake (NCREE, 2000).

Numerous studies have been conducted to understand the behavior of cohesionless soil under earthquake loading. Many of these studies have been based on the principle of subjecting representative soil elements to the same loading conditions in the laboratory, as they would encounter in the field, and assessing the probable field performance from the resulting behavior

Paper Received Oct. 9, 2001. Author for Correspondence: S. G. Chern. *Professor, Department of Harbor and River Engineering, National Taiwan Ocean University, Keelung, Taiwan, R.O.C.

**Graduate Student, Department of Harbor and River Engineering, National Taiwan Ocean University, Keelung, Taiwan, R.O.C. of the laboratory test specimens. Considerable amount of theoretical and empirical methods have also been developed for liquefaction evaluation.

Many of the existing assessment methods are developed from observations of the performance of sites during earthquakes (Seed et al., 1974; Tokimatsu et al., 1983; Robertson et al., 1992). Using field records like Standard Penetration Test blowcount SPT-N or Cone Penetration Test resistance CPT- $\mathrm{q}_{\mathrm{c}}$, empirical correlations are established between the soil and the seismic properties and the occurrence or nonoccurrence of liquefaction at the site.

The feasibility of using neural networks for assessing liquefaction potential from actual field records have been examined by many researchers (Goh, 1994; Juang, 2000; Wu, 1995). Neural networks are computer models whose architectures essentially mimic the biological system of the brain. Neural networks have been found to be useful for analyzing complex relationships involving a multitude of variables, in place of a conventional mathematical model. In neural networks, the mathematical relationship between the variables does not have to be specified. Instead, they learn from the examples and fed to them. An overview of neural network is presented, followed by a description of the proposed Fuzz-ART neural network model.

\section{OVERVIEW OF NEURAL NETWORKS}

A neural network consists of a number of interconnected proceeding units commonly referred to as neurodes or neurons. Each neuron receives an input signal from neurons to which it is connected. Each of these connections has numerical weights associated with it. These weights determine the nature and strength of the influence between the interconnected neurons. The signals from each input are then processed through a weighted sum of the inputs, and the processed output signal is then transmitted to another neuron via a transfer or activation function. A typical transfer function is the sigmoid transfer function. The sigmoid function modulates the weight sum of the inputs so that the 
output approaches unity when the input gets larger and approaches zero when the input gets smaller.

Fig. 1 shows the architecture of a typical neural network consisting of conventional three layers of interconnected neurons. Each neuron is connected to all the neurons in the next layer. There is an input layer that holds the response of the network to the input. It is the intermediate layers, also known as hidden layers that enable these networks to respect and compute complicated associations between patterns. A single hidden layer is common used in most conventional neural networks.

Training of the neural network is essentially carried out through the presentation of a series of example patterns of associated input and output values. The neural network learns what it is to compute through the modification of the weight of the interconnected neurons. Among many learning systems, back-propagation model is the most commonly used one. The learning algorithm processes the patterns in two stages. In the first stage, the input pattern generates a forward flow of signals from the input layer to the output layer. The error of each output neuron is then computed from the difference between the computed and the desired output. The second stage involves the readjustment of the weights in hidden and output layers to reduce the difference between the actual and desired output. Training is carried out iteratively until the average sum squared errors over all training patterns are minimized.

Once the training phase is computed satisfactorily, verification of the performance of the neural network is then carried out using patterns that were not included in the training set. This determines the quality of the predictions in comparison to the desired outputs. This is often called the testing phase. No additional learning

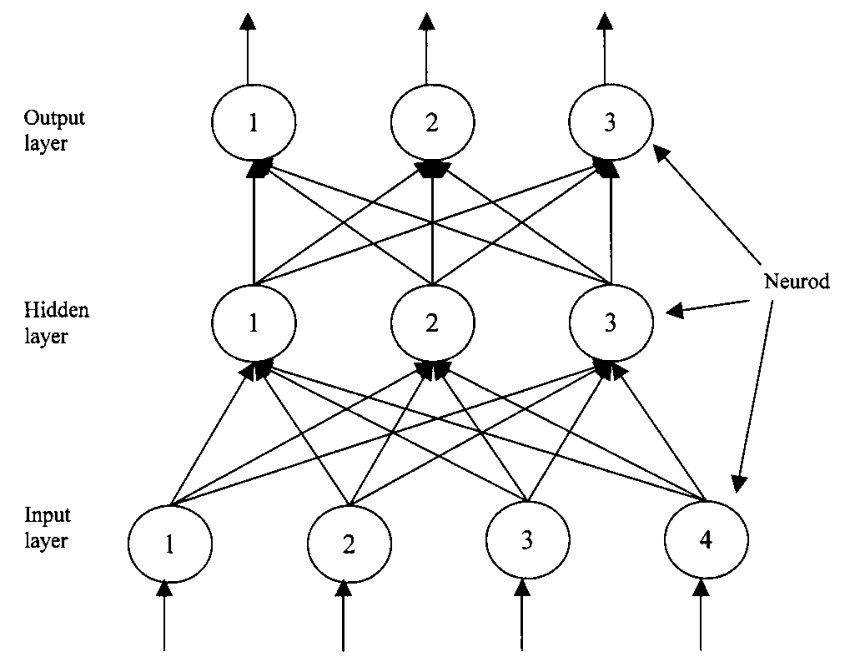

Fig. 1. Typical neural-network architecture. occurs during this phase.

Before presenting the input patterns to the neural network, some preprocessing of the data is necessary. This usually involves scaling or normalization of the input patterns to values in the range 0 to 1 . This is required because the sigmoid transfer function modulates the output to values between 0 and 1 .

\section{FUZZ-ART NEURAL NETWORK}

Based on adaptive resonance theory (ART) combined with fuzzy set theory, an adaptive network FuzzART is proposed to evaluate liquefaction potential. A five-layer consists of input, output and 3 hidden layers network topology, as shown in Fig. 2 is adopted in this study.

\section{The Structure of Fuzz-ART}

In the Fuzz-ART model, the technique of complement coding used in the fuzzy ART is adopted to normalize the input and output training vectors. This technique rescales an $\mathrm{n}$-dimensional vector in $R^{n}, x=$ $\left(x_{1}, x_{2}, \ldots, x_{n}\right)^{t}$, to its $2 n$-dimensional form in $[0,1]^{2 n}$ such that $x^{\prime}=\left(x_{1}^{\prime},\left(x_{1}^{\prime}\right)^{c}, x_{2}^{\prime},\left(x_{2}^{\prime}\right)^{c}, \ldots, x_{n}^{\prime},\left(x_{n}^{\prime}\right)^{c}\right)^{t} \equiv$ $\bar{x}=\left(\bar{x}_{1}, \bar{x}_{2}, \cdots \bar{x}_{n}\right)^{t}$, where $\bar{x}=\left(\bar{x}_{1}, \bar{x}_{2}, \cdots \bar{x}_{n}\right)^{t}=x$ / $\|x\|$, and $\mathrm{t}$ denotes the transpose operation of a vector or a matrix; all training vectors are transformed to their complement coded forms in the preprocessing process.

The functions of the nodes in each layer of the Fuzz-ART model are described as follows.

Layer 1:

There are two output values $x_{i}$ and $x_{i}^{c}=1-x_{i}$ due

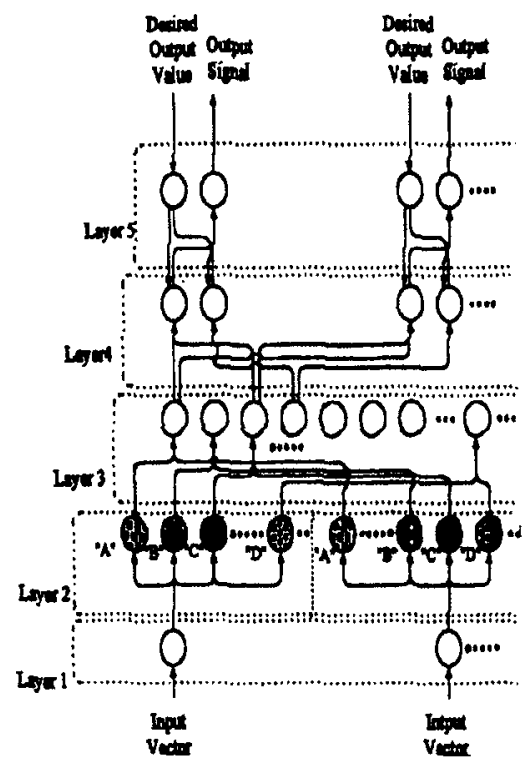

Fig. 2. Fuzz-ART neural-network architecture. 
to the complement coding for each input node $i$. The nodes in this layer transmit input signals to the next layer directly.

Layer 2:

Each node in this layer acts as membership function. The generalized bell membership function is used here. We denote the two weights on the link from input node as $u_{i j}$ and $v_{i j}^{c}$ that define the membership function. Layer3:

Each node in this layer represents one fuzzy logic rule and the links are used to perform precondition matching of fuzzy logic rules. This forms a multidimensional generalized bell membership function. Layer 4:

In this layer, each node has two operating modes, i.e. down-up transmission and up-down transmission modes. In down-up transmission mode, the links in Layer 4 perform the fuzzy OR operation. While in updown transmission mode, the nodes here and the links in Layer 5 function are the same as those in Layer 2. In other words, there are also two weighs on each up-down transmission links in Layer 5 to define hyperboxes in the output space.

Layer 5:

There are also two kinds of nodes in Layer 5. One is up-down transmission for training data to feed into the networks acting like Layer 1 . The other is down-up transmission for decision signal output. These nodes and the Layer 5 down-up transmission links attached to them act as a defuzzifier.

\section{Input Clustering Algorithm}

We use the fuzzy ART fast-learning algorithm to find the input membership function parameter.

1. Input vector:

Each input $I$ is an n-dimensional vector, where each component $I_{i}$ is in the interval $[0,1]$.

2. Choice function:

$$
T_{j}(I)=\frac{|I \wedge w|}{\alpha+\left|w_{j}\right|}, \text { where the fuzzy AND operator } \wedge
$$

is defined by $(x \wedge y)_{i} \equiv \min \left(x_{i}, y_{i}\right)$ and where the norm | $\mid$ is defined by $|x|=\sum_{i=1}^{M}\left|x_{i}\right|$

The category choice is indexed by $J$, where $T_{j}=$ $\max \left\{T_{j}: j=1 \ldots N\right\}$

3. Match function:

$$
\text { If } \frac{|I \wedge w|}{|I|} \geq \rho, \text { resonance will occur and if } \frac{\left|I \wedge w_{j}\right|}{|I|}
$$

$<\rho$, mismatch reset will occur.

Where $\rho$ is a vigilance parameter.

4. Weight update rule equation:
The weight vector is updated according to the equation

$$
w_{j}^{\text {new }}=\beta\left(I \wedge w_{j}^{\text {old }}\right)+(1-\beta) w_{j}^{\text {old }}
$$

Fast learning corresponds to setting $\beta=1$.

\section{Case Studies}

Comparison with existing neural network methods, two liquefaction examples taken from Goh (1994) and $\mathrm{Wu}$ (1995) where parameters of total and effective overburden pressure $\sigma_{0}$ and $\sigma_{0}$, , depth to groundwater table $\mathrm{Hw}$, relative density of sand Dr, fine content FC, maximum surface acceleration amax, SPT-N modification coefficient $\mathrm{Cn}$, magnitude of earthquake $\mathrm{M}$, earthquake induced shear stress $\tau_{a v}$, and stress reduction factor $\gamma_{d}$ are used to compare the present Fuzz-ART results with conventional three layer network results. The same training and testing data as used in Goh and Wu's networks are used for the proposed Fuzz-ART network. First the case records from Goh (1994) are evaluated using the Fuzz-ART neural networks. A total of 85 case records are considered. The data consists of 73 case records from Japan and 12 case records from the United States and Pan America, taken from 13 earthquakes that occurred in the period 1891-1980. This represented 42 sites that liquefied and 43 sites that did not liquefy. 59 of these case records are used for the training phase, and 26 for the testing phase. Details are shown in Table 1 and 2, respectively.

The results of the predications using this FuzzART would have been tabulated in Table 1 and 2 alongside the actual field performance and Goh's three layer neural network results. Altogether there is only one error in the training data also one error in testing data. In comparison, the Seed et al. (1985) procedure gave 14 errors or a $84 \%$ success rate (Goh, 1994), the Goh (1994) model gave 2 errors in the training data and 2 errors in testing data. This indicates that the present Fuzz-ART network approach has a much higher success rate $(98.3 \%$ success rate in training and $96.2 \%$ success rate in testing, overall $97.6 \%$ ) for evaluating liquefaction potential. Fig. 3 and 4 show variations of the magnitude of the errors at different stages of the Goh's conventional and the present Fuzz-ART neural network training phase. The difference in the average sum squared errors are minimal after about 20,000 cycles for Goh's model; however, they are minimal after only 100 cycles in the present Fuzz-ART model. It is found that the present Fuzz-ART model converges much quicker than Goh's model. The case records from Wu (1995) are listed in Table 3 and 4 . The results of the predications made by Wu (1995) and by using the present Fuzz-ART 
Table 1. The result of prediction for training set (Goh, 1994)

\begin{tabular}{|c|c|c|c|c|c|c|c|c|c|c|}
\hline \multirow[b]{2}{*}{ M } & \multirow[b]{2}{*}{$\sigma_{0}$} & \multirow[b]{2}{*}{$\sigma_{0}$} & \multirow[b]{2}{*}{$\mathrm{N}$} & \multirow[b]{2}{*}{$a / g$} & \multirow[b]{2}{*}{$\tau_{a v} / \sigma_{0}^{\prime}$} & \multirow[b]{2}{*}{$\mathrm{F}$} & \multirow[b]{2}{*}{$D_{50}$} & \multirow{2}{*}{$\begin{array}{c}\text { Field } \\
\text { behavior }\end{array}$} & \multicolumn{2}{|c|}{ The result of prediction } \\
\hline & & & & & & & & & $\begin{array}{c}\text { Reference } \\
\text { neural network }\end{array}$ & $\begin{array}{r}\text { Proposed } \\
\text { Fuzz-ART }\end{array}$ \\
\hline 7.9 & 186.4 & 96.1 & 20 & 0.32 & 0.36 & 0 & 0.46 & Yes & Yes & Yes \\
\hline 7.9 & 130.5 & 81.4 & 10 & 0.32 & 0.32 & 5 & 0.28 & Yes & Yes & Yes \\
\hline 7.9 & 111.8 & 71.6 & 17 & 0.28 & 0.28 & 3 & 0.80 & Yes & Yes & Yes \\
\hline 7.9 & 93.2 & 67.7 & 13 & 0.28 & 0.25 & 4 & 0.60 & Yes & Yes & Yes \\
\hline 7.9 & 122.6 & 93.2 & 10 & 0.20 & 0.16 & 10 & 0.25 & Yes & Yes & Yes \\
\hline 7.9 & 141.3 & 102.0 & 1 & 0.20 & 0.17 & 14 & 0.25 & Yes & Yes & Yes \\
\hline 7.9 & 71.6 & 69.7 & 2.2 & 0.20 & 0.13 & 22 & 0.18 & Yes & Yes & Yes \\
\hline 7.9 & 149.1 & 80.4 & 16.5 & 0.20 & 0.23 & 1 & 0.28 & Yes & Yes & Yes \\
\hline 7.9 & 93.2 & 63.8 & 11.9 & 0.20 & 0.19 & 5 & 0.30 & Yes & Yes & Yes \\
\hline 7.9 & 93.2 & 73.6 & 5.7 & 0.20 & 0.16 & 20 & 0.20 & No & No & No \\
\hline 7.9 & 149.1 & 100.1 & 2 & 0.20 & 0.18 & 33 & 0.15 & No & No & No \\
\hline 8 & 89.3 & 59.8 & 8 & 0.20 & 0.19 & 10 & 0.40 & Yes & Yes & Yes \\
\hline 8 & 64.7 & 35.3 & 1 & 0.20 & 0.24 & 27 & 0.20 & Yes & Yes & Yes \\
\hline 8 & 50.0 & 45.1 & 2 & 0.20 & 0.15 & 30 & 0.15 & Yes & Yes & Yes \\
\hline 8 & 130.5 & 81.4 & 10 & 0.16 & 0.16 & 5 & 0.28 & Yes & Yes & Yes \\
\hline 7.3 & 66.7 & 35.3 & 7 & 0.35 & 0.39 & 35 & 0.13 & No & No & No \\
\hline 7.3 & 141.3 & 70.6 & 29 & 0.35 & 0.39 & 2 & 0.80 & No & No & No \\
\hline 7.3 & 123.6 & 91.2 & 19 & 0.35 & 0.27 & 4 & 0.65 & Yes & Yes & Yes \\
\hline 7.3 & 74.6 & 47.1 & 8 & 0.40 & 0.38 & 0 & 0.45 & Yes & Yes & Yes \\
\hline 7.3 & 100.1 & 51.0 & 8 & 0.40 & 0.45 & 21 & 0.10 & No & No & No \\
\hline 7.3 & 128.5 & 63.8 & 20 & 0.40 & 0.45 & 0 & 0.45 & No & No & No \\
\hline 7.5 & 130.5 & 71.6 & 8 & 0.16 & 0.17 & 2 & 0.30 & Yes & Yes & Yes \\
\hline 7.5 & 130.5 & 71.6 & 12 & 0.16 & 0.17 & 2 & 0.30 & No & No & No \\
\hline 7.5 & 128.5 & 79.5 & 18 & 0.16 & 0.15 & 2 & 0.30 & No & No & No \\
\hline 7.5 & 186.4 & 98.1 & 10 & 0.16 & 0.17 & 2 & 0.30 & Yes & Yes & Yes \\
\hline 7.5 & 186.4 & 98.1 & 16 & 0.16 & 0.17 & 2 & 0.30 & No & No & No \\
\hline 7.5 & 186.4 & 105.9 & 20 & 0.16 & 0.15 & 2 & 0.30 & No & No & No \\
\hline 7.5 & 80.4 & 38.3 & 4 & 0.16 & 0.21 & 10 & 0.40 & Yes & Yes & Yes \\
\hline 7.5 & 111.8 & 65.7 & 27 & 0.16 & 0.16 & 0 & 0.30 & No & No & No \\
\hline 7.5 & 93.2 & 68.7 & 12 & 0.16 & 0.13 & 0 & 0.36 & No & No & No \\
\hline 7.5 & 84.4 & 46.1 & 6 & 0.16 & 0.18 & 0 & 0.40 & Yes & Yes & Yes \\
\hline 7.9 & 74.6 & 45.1 & 5 & 0.20 & 0.21 & 20 & 0.12 & Yes & Yes & Yes \\
\hline 7.9 & 111.8 & 72.6 & 28 & 0.23 & 0.22 & 5 & 0.25 & No & No & No \\
\hline 7.9 & 74.6 & 41.2 & 6 & 0.23 & 0.27 & 5 & 0.25 & Yes & Yes & Yes \\
\hline 7.9 & 74.6 & 45.1 & 16 & 0.23 & 0.25 & 5 & 0.25 & No & No & No \\
\hline 6.7 & 118.7 & 66.7 & 10 & 0.10 & 0.09 & 0 & 0.60 & No & No & No \\
\hline 6.7 & 61.8 & 34.3 & 5 & 0.12 & 0.12 & 5 & 0.70 & Yes & Yes & Yes \\
\hline 6.7 & 61.8 & 41.2 & 7 & 0.12 & 0.1 & 4 & 0.28 & No & No & No \\
\hline 6.7 & 80.4 & 47.1 & 11 & 0.12 & 0.11 & 0 & 0.40 & No & No & No \\
\hline 6.7 & 80.4 & 54.9 & 4 & 0.12 & 0.09 & 10 & 0.40 & No & No & No \\
\hline 6.7 & 61.8 & 41.2 & 13 & 0.12 & 0.10 & 7 & 1.60 & No & No & No \\
\hline 6.7 & 80.4 & 41.2 & 9 & 0.12 & 0.13 & 12 & 1.20 & No & No & No \\
\hline 6.7 & 103.0 & 83.4 & 9 & 0.14 & 0.09 & 5 & 0.34 & No & No & No \\
\hline 6.7 & 108.9 & 70.6 & 8 & 0.14 & 0.11 & 4 & 0.36 & No & No & No \\
\hline 6.7 & 59.8 & 56.9 & 11 & 0.14 & 0.08 & 5.0 & 0.53 & No & No & No \\
\hline 6.7 & 74.6 & 59.8 & 6 & 0.14 & 0.09 & 10 & 0.25 & No & No & No \\
\hline 6.7 & 93.2 & 68.7 & 9 & 0.14 & 0.10 & 20 & 0.15 & No & No & No \\
\hline 6.7 & 111.8 & 77.5 & 12 & 0.14 & 0.11 & 3 & 0.35 & No & No & No \\
\hline 6.7 & 74.6 & 49.1 & 4 & 0.12 & 0.10 & 10 & 0.15 & No & No & No \\
\hline 5.5 & 111.8 & 48.1 & 6 & 0.19 & 0.10 & 3 & 0.20 & No & No & No \\
\hline 8.3 & 56.9 & 53.0 & 10 & 0.16 & 0.22 & 10 & 0.20 & Yes & Yes & Yes \\
\hline 6.6 & 72.6 & 86.3 & 9 & 0.45 & 0.29 & 20 & 0.10 & Yes & Yes & Yes \\
\hline 7.5 & 72.6 & 28.4 & 8 & 0.14 & 0.17 & 3 & 1.00 & Yes & Yes & Yes \\
\hline 7.5 & 93.2 & 34.3 & 8 & 0.14 & 0.14 & 3 & 1.00 & Yes & Yes & Yes \\
\hline 7.5 & 58.9 & 62.8 & 14 & 0.14 & 0.13 & 3 & 1.00 & No & Yes & Yes \\
\hline 6.6 & 107.9 & 51.0 & 31 & 0.60 & 0.45 & 11 & 0.12 & No & No & No \\
\hline 6.6 & 58.9 & 51.0 & 4 & 0.60 & 0.45 & 25 & 0.12 & No & No & No \\
\hline 6.6 & 686.7 & 51.0 & 11 & 0.60 & 0.45 & 19 & 0.10 & Yes & Yes & Yes \\
\hline 6.6 & 72.6 & 46.1 & 7 & 0.20 & 0.21 & 34 & 0.09 & No & No & No \\
\hline 7.4 & 118.7 & 66.7 & 10 & 0.20 & 0.21 & 0 & 0.60 & Yes & No & Yes \\
\hline
\end{tabular}

The shadow determinates the error of prediction 
Table 2. The result of prediction for testing set (Goh, 1994)

\begin{tabular}{|c|c|c|c|c|c|c|c|c|c|c|}
\hline \multirow[b]{2}{*}{ M } & \multirow[b]{2}{*}{$\sigma_{0}$} & \multirow[b]{2}{*}{$\sigma_{0}$} & \multirow[b]{2}{*}{$\mathrm{N}$} & \multirow[b]{2}{*}{$a / g$} & \multirow[b]{2}{*}{$\tau_{a v} / \sigma_{0}^{\prime}$} & \multirow[b]{2}{*}{$\mathrm{F}$} & \multirow[b]{2}{*}{$D_{50}$} & \multirow{2}{*}{$\begin{array}{c}\text { Field } \\
\text { behavior }\end{array}$} & \multicolumn{2}{|c|}{ The result of prediction } \\
\hline & & & & & & & & & $\begin{array}{c}\text { Reference } \\
\text { neural network }\end{array}$ & $\begin{array}{c}\text { Proposed } \\
\text { Fuzz-ART }\end{array}$ \\
\hline 7.4 & 118.7 & 66.7 & 10 & 0.20 & 0.21 & 0 & 0.60 & Yes & Yes & Yes \\
\hline 7.4 & 61.8 & 38.3 & 19 & 0.32 & 0.31 & 4 & 0.28 & No & No & No \\
\hline 7.4 & 61.8 & 34.3 & 5 & 0.32 & 0.35 & 5 & 0.70 & Yes & Yes & Yes \\
\hline 7.4 & 61.8 & 41.2 & 7 & 0.32 & 0.29 & 4 & 0.28 & Yes & Yes & Yes \\
\hline 7.4 & 80.4 & 47.1 & 11 & 0.24 & 0.25 & 0 & 0.40 & Yes & Yes & Yes \\
\hline 7.4 & 97.1 & 66.7 & 20 & 0.24 & 0.21 & 0 & 0.60 & No & No & No \\
\hline 7.4 & 80.4 & 54.9 & 4 & 0.24 & 0.21 & 10 & 0.40 & Yes & Yes & Yes \\
\hline 7.4 & 61.8 & 41.2 & 13 & 0.24 & 0.22 & 7 & 1.60 & Yes & Yes & Yes \\
\hline 7.4 & 80.4 & 41.2 & 8 & 0.24 & 0.28 & 12 & 1.20 & Yes & Yes & Yes \\
\hline 7.4 & 136.4 & 77.5 & 17 & 0.24 & 0.24 & 17 & 0.35 & No & No & No \\
\hline 7.4 & 103.0 & 83.4 & 9 & 0.24 & 0.17 & 5 & 0.34 & Yes & Yes & Yes \\
\hline 7.4 & 108.9 & 70.6 & 8 & 0.24 & 0.21 & 4 & 0.36 & Yes & Yes & Yes \\
\hline 7.4 & 9.8 & 56.9 & 11 & 0.28 & 0.18 & 5 & 0.53 & Yes & Yes & Yes \\
\hline 7.4 & 109.9 & 80.4 & 23 & 0.28 & 0.22 & 0 & 0.41 & No & No & No \\
\hline 7.4 & 111.8 & 77.5 & 10 & 0.24 & 0.20 & 10 & 0.30 & No & Yes & Yes \\
\hline 7.4 & 74.6 & 59.8 & 6 & 0.24 & 0.18 & 10 & 0.25 & Yes & Yes & Yes \\
\hline 7.4 & 130.5 & 86.3 & 21 & 0.24 & 0.21 & 5 & 0.35 & No & No & No \\
\hline 7.4 & 93.2 & 68.7 & 9 & 0.24 & 0.19 & 20 & 0.15 & Yes & No & Yes \\
\hline 7.4 & 83.4 & 63.8 & 10 & 0.24 & 0.19 & 26 & 0.12 & No & No & No \\
\hline 7.4 & 111.8 & 77.5 & 12 & 0.24 & 0.20 & 3 & 0.35 & Yes & Yes & Yes \\
\hline 7.4 & 106.9 & 71.6 & 15 & 0.24 & 0.21 & 11 & 0.30 & No & No & No \\
\hline 7.4 & 124.6 & 91.2 & 17 & 0.24 & 0.19 & 12 & 0.30 & No & No & No \\
\hline 7.4 & 74.6 & 49.1 & 4 & 0.20 & 0.18 & 10 & 0.15 & Yes & Yes & Yes \\
\hline 7.4 & 111.8 & 66.7 & 15 & 0.20 & 0.20 & 10 & 0.18 & No & No & No \\
\hline 6.1 & 105.9 & 56.9 & 5 & 0.10 & 0.09 & 13 & 0.18 & No & No & No \\
\hline 6.1 & 247.2 & 105.9 & 4 & 0.10 & 0.09 & 27 & 0.17 & No & No & No \\
\hline
\end{tabular}

The shadow determinates the error of prediction

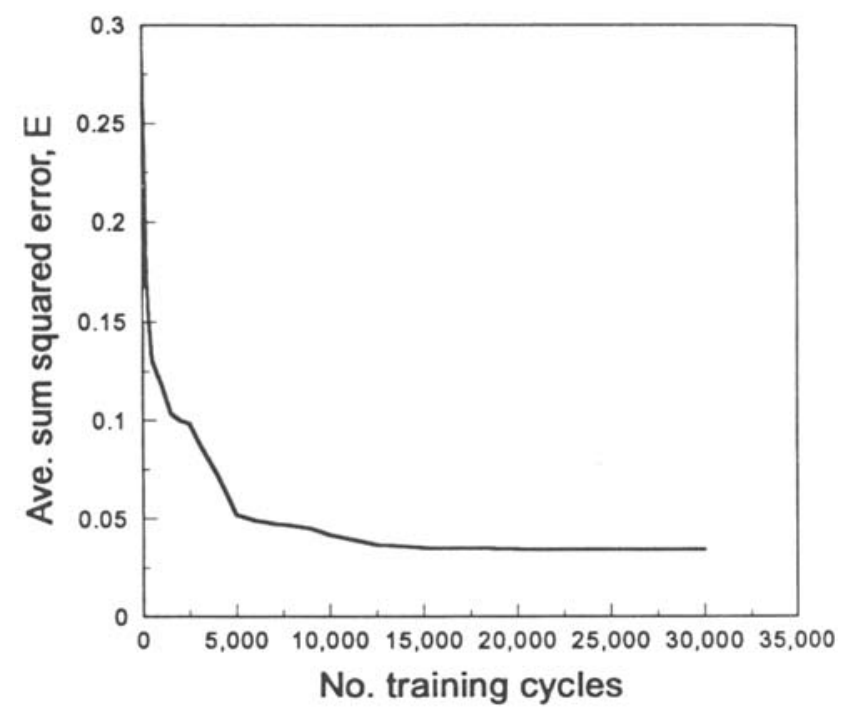

Fig. 3. Learning curve of reference neural network (Goh, 1994).

model are tabulated in Table 3 alongside the actual field performance. The success rates of the Wu's and FuzzART neural networks are summarized in Table 5. The results indicate that the Fuzz-ART neural network is

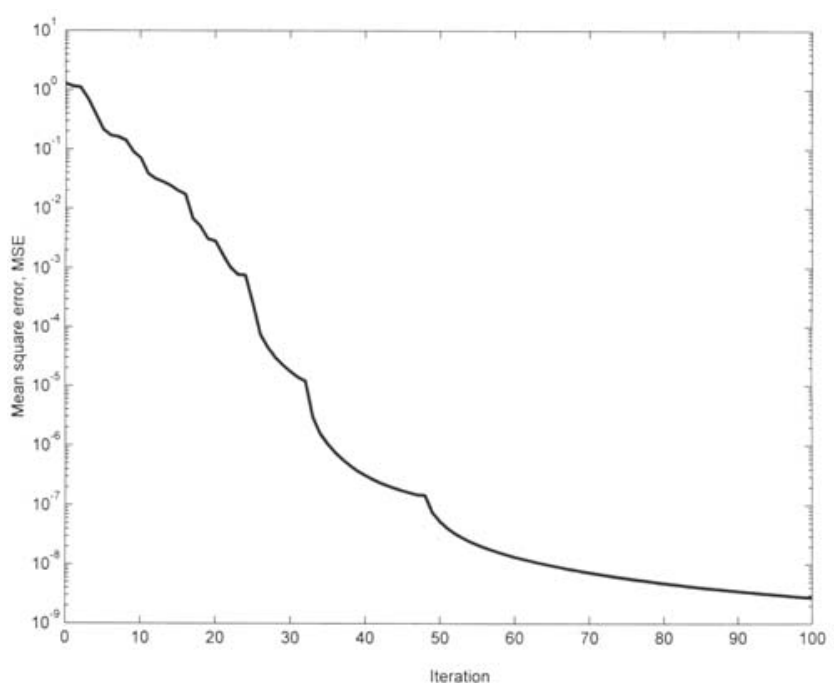

Fig. 4. Learning curve of Fuzz-ART.

more successful in learning the relationship between the input and output data than Wu's conventional 3 layer neural network model. The results from the testing phase suggest that the Fuzz-ART neural network gives more reasonable predications than conventional one. 
Table 3. Field behavior data for training set $(\mathrm{Wu}, 1995)$

(1: liquefaction $\quad 0$ : no liquefaction)

\begin{tabular}{|c|c|c|c|c|c|c|c|c|c|c|c|}
\hline Dcr & $\mathrm{Hw}$ & $\tau_{a v} / \sigma_{0}^{\prime}$ & $\gamma_{d}$ & $D_{50}$ & $\mathrm{~N}$ & Dr & $\mathrm{Cn}$ & $a_{\max }$ & $\begin{array}{c}\text { Field } \\
\text { behavior }\end{array}$ & $\begin{array}{c}\text { Reference } \\
\text { model }\end{array}$ & $\begin{array}{c}\text { Fuzz-ART } \\
\text { model }^{*}\end{array}$ \\
\hline 10.1 & 0.9 & 1.90 & 0.90 & 0.46 & 20 & 72.61 & 0.98 & 0.32 & 1 & 0.758 & 0.096 \\
\hline 7 & 2.1 & 1.57 & 0.95 & 0.28 & 10 & 60.12 & 1.06 & 0.32 & 1 & 1.004 & 0.551 \\
\hline 6.1 & 1.8 & 1.57 & 0.96 & 0.80 & 17 & 70.14 & 1.13 & 0.32 & 1 & 1.010 & 0.768 \\
\hline 5.2 & 2.4 & 1.37 & 0.96 & 0.60 & 13 & 65.04 & 1.15 & 0.32 & 1 & 0.931 & 0.851 \\
\hline 7 & 4.0 & 1.32 & 0.95 & 0.25 & 10 & 59.50 & 1.00 & 0.20 & 1 & 0.681 & 0.884 \\
\hline 4.3 & 4.0 & 1.04 & 0.97 & 0.18 & 2 & 47.35 & 1.14 & 0.20 & 1 & 0.768 & 1.030 \\
\hline 8.2 & 0.9 & 1.86 & 0.93 & 0.28 & 16 & 68.42 & 1.07 & 0.20 & 1 & 0.575 & 1.080 \\
\hline 5.2 & 0.9 & 1.74 & 0.96 & 0.30 & 12 & 64.44 & 1.28 & 0.20 & 1 & 0.729 & 1.090 \\
\hline 5.2 & 3.0 & 1.28 & 0.96 & 0.20 & 6 & 54.26 & 1.1 & 0.20 & 0 & 0.649 & 0.184 \\
\hline 8.2 & 1.8 & 1.68 & 0.93 & 0.11 & 8 & 56.82 & 1.03 & 0.20 & 0 & 1.151 & 0.273 \\
\hline 6.1 & 1.8 & 1.56 & 0.95 & 0.44 & 7 & 56.30 & 1.16 & 0.16 & 0 & 0.526 & 0.251 \\
\hline 5.2 & 2.1 & 1.44 & 0.96 & 0.40 & 8 & 57.98 & 1.17 & 0.20 & 1 & 0.533 & 0.904 \\
\hline 3.7 & 0.9 & 1.76 & 0.98 & 0.20 & 1 & 47.68 & 1.49 & 0.20 & 1 & 0.964 & 1.070 \\
\hline 3 & 2.4 & 1.12 & 0.98 & 0.15 & 2 & 49.00 & 1.37 & 0.20 & 1 & 1.078 & 1.050 \\
\hline 7 & 2.1 & 1.57 & 0.95 & 0.28 & 10 & 60.12 & 1.06 & 0.16 & 1 & 0.327 & 0.858 \\
\hline 8.2 & 0.9 & 1.88 & 0.93 & 0.80 & 29 & 83.27 & 1.08 & 0.35 & 0 & -0.173 & 0.096 \\
\hline 7 & 3.7 & 1.33 & 0.95 & 0.65 & 19 & 71.45 & 0.98 & 0.35 & 1 & 1.036 & 0.729 \\
\hline 4 & 1.2 & 1.56 & 0.97 & 0.45 & 8 & 59.10 & 1.37 & 0.40 & 1 & 1.213 & 0.909 \\
\hline 7.6 & 0.9 & 1.89 & 0.94 & 0.45 & 20 & 73.74 & 1.12 & 0.40 & 0 & 0.364 & 0.096 \\
\hline 3 & 2.4 & 1.13 & 0.98 & 0.34 & 5 & 54.48 & 1.40 & 0.19 & 1 & 0.829 & 0.977 \\
\hline 7 & 0.9 & 1.83 & 0.95 & 0.30 & 8 & 57.74 & 1.14 & 0.16 & 1 & 0.812 & 0.878 \\
\hline 7 & 0.9 & 1.83 & 0.95 & 0.30 & 12 & 63.62 & 1.14 & 0.16 & 0 & 0.475 & 0.097 \\
\hline 7 & 1.8 & 1.62 & 0.95 & 0.30 & 18 & 71.01 & 1.00 & 0.18 & 0 & -0.144 & 0.096 \\
\hline 10.1 & 0.9 & 1.90 & 0.91 & 0.30 & 10 & 59.26 & 0.98 & 0.16 & 1 & 0.789 & 0.834 \\
\hline 10.1 & 0.9 & 1.90 & 0.91 & 0.30 & 16 & 67.63 & 0.98 & 0.16 & 0 & 0.292 & 0.096 \\
\hline 10.1 & 1.8 & 1.74 & 0.91 & 0.30 & 20 & 72.24 & 0.94 & 0.18 & 0 & -0.129 & 0.096 \\
\hline 4.3 & 0.0 & 2.07 & 0.97 & 0.40 & 4 & 53.20 & 1.50 & 0.18 & 1 & 0.768 & 1.090 \\
\hline 6.1 & 1.2 & 1.71 & 0.96 & 0.30 & 27 & 81.76 & 1.18 & 0.18 & 0 & -0.214 & 0.096 \\
\hline 6.1 & 2.4 & 1.46 & 0.96 & 0.30 & 12 & 63.28 & 1.09 & 0.18 & 0 & 0.034 & 0.096 \\
\hline 4.6 & 0.6 & 1.82 & 0.97 & 0.40 & 6 & 56.00 & 1.38 & 0.16 & 1 & 0.964 & 1.090 \\
\hline 0.9 & 0.9 & 1.00 & 0.99 & 0.15 & 3 & 52.91 & 1.98 & 0.13 & 1 & 1.040 & 1.090 \\
\hline 4 & 0.9 & 1.66 & 0.97 & 0.12 & 5 & 54.50 & 1.41 & 0.2 & 1 & 0.936 & 1.090 \\
\hline 6.1 & 2.1 & 1.51 & 0.96 & 0.25 & 28 & 82.46 & 1.10 & 0.23 & 0 & -0.204 & 0.096 \\
\hline 4 & 0.6 & 1.79 & 0.97 & 0.25 & 6 & 56.34 & 1.47 & 0.23 & 1 & 1.134 & 0.950 \\
\hline 4 & 0.9 & 1.66 & 0.97 & 0.25 & 16 & 70.17 & 1.41 & 0.23 & 0 & 0.149 & 0.096 \\
\hline 6.1 & 3.0 & 1.25 & 0.95 & 0.10 & 9 & 58.51 & 1.05 & 0.45 & 1 & 0.778 & 1.090 \\
\hline 6.1 & 4.6 & 1.17 & 0.95 & 0.08 & 2 & 45.56 & 1.00 & 0.45 & 1 & 1.153 & 1.010 \\
\hline 6 & 2.0 & 1.55 & 0.96 & 0.10 & 9.5 & 60.40 & 1.14 & 0.10 & 0 & 0.152 & 0.096 \\
\hline 13 & 2.0 & 1.74 & 0.83 & 0.10 & 14.5 & 63.40 & 0.81 & 0.10 & 0 & 0.066 & 0.096 \\
\hline 6.2 & 1.5 & 1.62 & 0.96 & 0.12 & 6 & 54.40 & 1.12 & 0.10 & 1 & 0.399 & 1.060 \\
\hline 3 & 2.0 & 1.20 & 0.98 & 0.12 & 6 & 56.03 & 1.40 & 0.13 & 1 & 0.337 & 1.090 \\
\hline 10 & 2.0 & 1.68 & 0.91 & 0.12 & 9 & 56.82 & 0.91 & 0.20 & 1 & 1.171 & 1.010 \\
\hline 10.3 & 2.0 & 1.69 & 0.90 & 0.12 & 9 & 56.66 & 0.89 & 0.20 & 1 & 1.172 & 1.000 \\
\hline 9.1 & 1.5 & 1.74 & 0.92 & 0.06 & 8 & 56.07 & 0.98 & 0.13 & 1 & 0.871 & 1.090 \\
\hline 8.2 & 1.5 & 1.71 & 0.93 & 0.09 & 11 & 61.16 & 1.02 & 0.20 & 1 & 1.127 & 1.090 \\
\hline 8.2 & 1.5 & 1.71 & 0.93 & 0.08 & 13 & 63.99 & 1.02 & 0.20 & 1 & 1.077 & 1.090 \\
\hline 8.2 & 1.5 & 1.71 & 0.93 & 0.08 & 9 & 58.16 & 1.02 & 0.10 & 0 & 0.462 & 0.096 \\
\hline 2.3 & 1.4 & 1.28 & 0.98 & 0.12 & 11 & 64.22 & 1.62 & 0.20 & 1 & 0.645 & 1.090 \\
\hline 7 & 1.0 & 1.82 & 0.94 & 0.12 & 4 & 51.05 & 1.14 & 0.35 & 1 & 1.203 & 0.958 \\
\hline 5.3 & 3.0 & 1.30 & 0.96 & 0.20 & 30 & 84.54 & 1.12 & 0.50 & 0 & -0.031 & 0.096 \\
\hline 5.3 & 0.9 & 1.73 & 0.96 & 0.14 & 17 & 70.79 & 1.25 & 0.35 & 1 & 0.696 & 1.090 \\
\hline 5.4 & 3.7 & 1.21 & 0.96 & 0.17 & 20 & 73.48 & 1.09 & 0.22 & 1 & 0.559 & 1.090 \\
\hline 2 & 1.5 & 1.16 & 0.99 & 0.22 & 10 & 62.90 & 1.65 & 0.20 & 1 & 0.920 & 1.090 \\
\hline
\end{tabular}


Table 3. Field behavior data for training set $(\mathrm{Wu}, 1995)$ (Continue)

(1: liquefaction $\quad 0$ : no liquefaction)

\begin{tabular}{|c|c|c|c|c|c|c|c|c|c|c|c|}
\hline Dcr & $\mathrm{Hw}$ & $\tau_{a v} / \sigma_{0}^{\prime}$ & $\gamma_{d}$ & $D_{50}$ & $\mathrm{~N}$ & Dr & $\mathrm{Cn}$ & $a_{\max }$ & $\begin{array}{c}\text { Field } \\
\text { behavior }\end{array}$ & $\begin{array}{c}\text { Reference } \\
\text { model }\end{array}$ & $\begin{array}{c}\text { Fuzz-ART } \\
\text { model* }\end{array}$ \\
\hline 6.1 & 1.2 & 1.69 & 0.96 & 0.14 & 10 & 60.94 & 1.10 & 0.13 & 1 & 0.609 & 1.090 \\
\hline 6.1 & 0.9 & 1.76 & 0.90 & 0.09 & 9 & 59.58 & 1.19 & 0.20 & 1 & 0.871 & 1.090 \\
\hline 9.1 & 4.0 & 1.43 & 0.92 & 0.30 & 13 & 62.62 & 0.90 & 0.07 & 0 & -0.198 & 0.096 \\
\hline 7 & 1.5 & 1.67 & 0.94 & 0.32 & 11 & 61.80 & 1.06 & 0.20 & 1 & 0.813 & 0.821 \\
\hline 10.4 & 1.5 & 1.62 & 0.89 & 0.80 & 6 & 53.67 & 1.05 & 0.14 & 1 & 0.955 & 0.907 \\
\hline 4.6 & 2.4 & 1.63 & 0.96 & 0.80 & 8 & 59.87 & 1.60 & 0.14 & 0 & -0.128 & 0.097 \\
\hline 11.6 & 2.4 & 1.73 & 0.86 & 0.80 & 15 & 66.85 & 1.03 & 0.14 & 0 & -0.214 & 0.096 \\
\hline 10.7 & 4.3 & 1.92 & 0.89 & 0.80 & 16 & 68.97 & 1.14 & 0.14 & 0 & -0.214 & 0.096 \\
\hline 8.2 & 4.6 & 1.34 & 0.94 & 0.15 & 9 & 57.37 & 0.95 & 0.20 & 1 & 0.948 & 1.090 \\
\hline 11.9 & 6.7 & 1.50 & 0.85 & 0.15 & 12 & 62.10 & 0.97 & 0.20 & 1 & 0.977 & 1.090 \\
\hline 3.7 & 1.2 & 1.61 & 0.97 & 0.39 & 14 & 67.90 & 1.50 & 0.20 & 0 & 0.115 & 0.097 \\
\hline 3 & 2.1 & 1.21 & 0.98 & 0.24 & 14 & 67.70 & 1.44 & 0.20 & 0 & -0.122 & 0.097 \\
\hline 5.2 & 1.8 & 1.59 & 0.90 & 0.08 & 6 & 55.47 & 1.28 & 0.20 & 1 & 0.894 & 1.090 \\
\hline 6.4 & 0.9 & 1.80 & 0.95 & 0.60 & 10 & 61.04 & 1.18 & 0.10 & 0 & -0.083 & 0.098 \\
\hline 3.4 & 0.6 & 1.74 & 0.98 & 0.70 & 5 & 55.02 & 1.55 & 0.12 & 1 & 0.960 & 1.090 \\
\hline 3.4 & 1.2 & 1.50 & 0.98 & 0.28 & 7 & 57.89 & 1.46 & 0.12 & 0 & 0.189 & 0.098 \\
\hline 5.5 & 1.8 & 1.57 & 0.96 & 0.04 & 2 & 47.96 & 1.22 & 0.12 & 0 & 0.167 & 0.096 \\
\hline 4.3 & 0.9 & 1.70 & 0.97 & 0.40 & 11 & 63.49 & 1.38 & 0.12 & 0 & -0.085 & 0.096 \\
\hline 4.3 & 1.8 & 1.42 & 0.97 & 0.40 & 4 & 52.08 & 1.27 & 0.12 & 0 & 0.263 & 0.096 \\
\hline 3.4 & 1.2 & 1.59 & 0.98 & 1.00 & 13 & 66.58 & 1.4 & 0.12 & 0 & -0.038 & 0.096 \\
\hline 4.3 & 0.3 & 1.93 & 0.97 & 1.20 & 8 & 59.44 & 1.47 & 0.12 & 0 & 0.032 & 0.096 \\
\hline 6.4 & 4.3 & 1.25 & 0.95 & 0.34 & 9 & 58.56 & 1.05 & 0.14 & 0 & 0.152 & 0.096 \\
\hline 6.4 & 2.4 & 1.51 & 0.95 & 0.36 & 8 & 57.55 & 1.11 & 0.14 & 0 & -0.025 & 0.104 \\
\hline 3.4 & 3.0 & 1.05 & 0.98 & 0.53 & 11 & 62.88 & 1.24 & 0.14 & 0 & 0.115 & 0.096 \\
\hline 4 & 2.4 & 1.25 & 0.97 & 0.25 & 6 & 55.27 & 1.24 & 0.14 & 0 & 0.105 & 0.096 \\
\hline 5.2 & 2.4 & 1.38 & 0.96 & 0.15 & 9 & 59.35 & 1.15 & 0.14 & 0 & 0.195 & 0.097 \\
\hline 6.1 & 2.4 & 1.46 & 0.96 & 0.35 & 12 & 63.28 & 1.09 & 0.14 & 0 & -0.144 & 0.099 \\
\hline 4 & 1.5 & 1.47 & 0.97 & 0.15 & 4 & 52.44 & 1.34 & 0.12 & 0 & 0.288 & 0.105 \\
\hline 6.4 & 0.9 & 1.80 & 0.95 & 0.60 & 10 & 61.04 & 1.18 & 0.20 & 1 & 1.125 & 0.911 \\
\hline 3.4 & 0.9 & 1.61 & 0.98 & 0.28 & 19 & 74.04 & 1.49 & 0.32 & 0 & 0.000 & 0.096 \\
\hline 3.4 & 0.6 & 1.74 & 0.98 & 0.70 & 5 & 55.02 & 1.55 & 0.32 & 1 & 1.214 & 1.080 \\
\hline 3.4 & 1.2 & 1.50 & 0.98 & 0.28 & 7 & 57.89 & 1.46 & 0.32 & 1 & 1.210 & 0.962 \\
\hline 3.7 & 1.8 & 1.36 & 0.97 & 0.11 & 28 & 83.45 & 1.35 & 0.78 & 0 & -0.207 & 0.096 \\
\hline 3.7 & 1.8 & 1.36 & 0.97 & 0.12 & 1 & 46.94 & 1.35 & 0.78 & 1 & 1.213 & 1.070 \\
\hline 4.3 & 1.8 & 1.43 & 0.96 & 0.09 & 13 & 65.81 & 1.28 & 0.78 & 0 & -0.054 & 0.096 \\
\hline 1.8 & 0.3 & 1.73 & 0.99 & 0.04 & 3 & 52.63 & 1.88 & 0.24 & 1 & 0.828 & 1.090 \\
\hline 4.3 & 0.3 & 1.87 & 0.96 & 0.15 & 11 & 63.63 & 1.42 & 0.24 & 1 & 0.807 & 1.090 \\
\hline 3.4 & 2.1 & 1.25 & 0.96 & 0.05 & 2 & 48.93 & 1.36 & 0.20 & 1 & 1.132 & 1.090 \\
\hline 2.3 & 2.1 & 1.04 & 0.98 & 0.10 & 11 & 63.95 & 1.51 & 0.20 & 0 & 0.240 & 0.096 \\
\hline 2.1 & 1.5 & 1.19 & 0.99 & 0.11 & 3 & 51.86 & 1.62 & 0.51 & 1 & 1.214 & 1.090 \\
\hline 4.9 & 0.9 & 1.70 & 0.97 & 0.09 & 9 & 60.21 & 1.29 & 0.26 & 1 & 1.121 & 1.090 \\
\hline 4.3 & 2.7 & 1.24 & 0.97 & 0.05 & 5 & 53.43 & 1.22 & 0.32 & 1 & 1.089 & 1.090 \\
\hline 1.8 & 0.3 & 1.73 & 0.99 & 0.04 & 3 & 52.63 & 1.88 & 0.21 & 0 & 0.229 & 0.101 \\
\hline 4.3 & 0.3 & 1.87 & 0.96 & 0.15 & 11 & 63.63 & 1.42 & 0.21 & 0 & 0.439 & 0.100 \\
\hline 3.4 & 2.1 & 1.25 & 0.94 & 0.05 & 2 & 48.93 & 1.46 & 0.2 & 1 & 1.081 & 1.090 \\
\hline 2.3 & 2.1 & 1.04 & 0.98 & 0.10 & 11 & 63.95 & 1.51 & 0.2 & 0 & 0.240 & 0.096 \\
\hline 2.1 & 1.5 & 1.19 & 0.99 & 0.11 & 3 & 51.86 & 1.62 & 0.09 & 0 & -0.035 & 0.098 \\
\hline
\end{tabular}

Fig. 5 and 6 show variations of the magnitude of the errors at different stage of Wu's conventional and the proposed Fuzz-ART neural networks. The differ- ence in the root mean square errors are minimal after about 10,000 cycles for Wu's model; however, they are minimal after only 8 cycles in the proposed Fuzz-ART 
Table 4. Field behavior data for testing set $(\mathrm{Wu}, 1995)$

\begin{tabular}{|c|c|c|c|c|c|c|c|c|c|c|c|}
\hline Dcr & $\mathrm{Hw}$ & $\tau_{a v} / \sigma_{0}^{\prime}$ & $\gamma_{d}$ & $D_{50}$ & $\mathrm{~N}$ & Dr & $\mathrm{Cn}$ & $a_{\max }$ & $\begin{array}{c}\text { Field } \\
\text { behavior }\end{array}$ & $\begin{array}{c}\text { Reference } \\
\text { model }\end{array}$ & $\begin{array}{c}\text { Fuzz-ART } \\
\text { model* }\end{array}$ \\
\hline 4.6 & 2.4 & 1.32 & 0.97 & 0.12 & 10 & 61.14 & 1.20 & 0.24 & 0 & 0.569 & 0.097 \\
\hline 6.1 & 2.4 & 1.46 & 0.96 & 0.35 & 12 & 63.28 & 1.09 & 0.24 & 1 & 0.289 & 0.808 \\
\hline 6.1 & 2.4 & 1.48 & 0.96 & 0.30 & 15 & 67.51 & 1.11 & 0.24 & 0 & 0.058 & 0.115 \\
\hline 7.0 & 3.7 & 1.35 & 0.95 & 0.30 & 17 & 69.18 & 1.00 & 0.24 & 0 & 0.631 & 0.105 \\
\hline 4.0 & 1.5 & 1.47 & 0.97 & 0.15 & 4 & 52.44 & 1.34 & 0.20 & 1 & 1.122 & 1.080 \\
\hline 6.1 & 1.5 & 1.64 & 0.96 & 0.18 & 15 & 67.76 & 1.16 & 0.20 & 0 & 0.262 & 0.096 \\
\hline 5.5 & 1.8 & 1.57 & 0.90 & 0.04 & 2 & 48.96 & 1.22 & 0.24 & 1 & 1.124 & 1.090 \\
\hline 4.3 & 1.8 & 1.7 & 0.97 & 0.40 & 11 & 60.49 & 1.38 & 0.24 & 1 & 1.132 & 0.814 \\
\hline 5.5 & 2.1 & 1.47 & 0.9 & 0.60 & 20 & 73.89 & 1.15 & 0.24 & 0 & -0.147 & 0.096 \\
\hline 4.3 & 1.8 & 1.42 & 0.97 & 0.40 & 4 & 52.08 & 1.27 & 0.24 & 1 & 1.195 & 0.511 \\
\hline 3.4 & 1.2 & 1.50 & 0.98 & 1.60 & 13 & 66.46 & 1.46 & 0.24 & 1 & 1.076 & 0.135 \\
\hline 4.3 & 0.3 & 1.93 & 0.97 & 1.20 & 8 & 59.44 & 1.47 & 0.24 & 1 & 1.195 & 0.558 \\
\hline 7.3 & 1.2 & 1.77 & 0.94 & 0.35 & 17 & 69.92 & 1.10 & 0.24 & 0 & 0.235 & 0.121 \\
\hline 6.4 & 4.3 & 1.25 & 0.95 & 0.34 & 9 & 58.56 & 1.05 & 0.24 & 1 & 0.951 & 0.547 \\
\hline 6.1 & 0.9 & 1.88 & 0.96 & 0.18 & 5 & 53.74 & 1.26 & 0.10 & 0 & -0.169 & 0.250 \\
\hline 6.1 & 0.9 & 1.88 & 0.96 & 0.18 & 5 & 53.74 & 1.26 & 0.11 & 0 & 0.127 & 0.250 \\
\hline 14.3 & 0.9 & 2.07 & 0.79 & 0.17 & 4 & 47.62 & 0.88 & 0.10 & 0 & -0.160 & 0.096 \\
\hline 14.3 & 0.9 & 2.07 & 0.79 & 0.17 & 4 & 47.62 & 0.88 & 0.15 & 0 & -0.003 & 0.096 \\
\hline 6.4 & 2.4 & 1.51 & 0.95 & 0.36 & 8 & 57.55 & 1.11 & 0.24 & 1 & 0.837 & 0.546 \\
\hline 3.4 & 3.0 & 1.05 & 0.98 & 0.53 & 11 & 62.88 & 1.24 & 0.28 & 1 & 1.102 & 0.607 \\
\hline 6.1 & 3.0 & 1.35 & 0.96 & 0.41 & 23 & 76.71 & 1.05 & 0.24 & 0 & 0.068 & 0.096 \\
\hline 6.1 & 2.4 & 1.43 & 0.96 & 0.30 & 10 & 60.33 & 1.08 & 0.24 & 0 & 0.548 & 0.138 \\
\hline 4.0 & 2.4 & 1.25 & 0.97 & 0.25 & 6 & 59.27 & 1.24 & 0.24 & 1 & 0.899 & 0.500 \\
\hline 7.0 & 2.4 & 1.52 & 0.95 & 0.35 & 21 & 74.34 & 1.04 & 0.24 & 0 & -0.097 & 0.096 \\
\hline 5.2 & 2.4 & 1.38 & 0.96 & 0.15 & 9 & 59.35 & 1.15 & 0.24 & 1 & 0.801 & 0.659 \\
\hline 2.2 & 1.6 & 1.17 & 0.99 & 0.22 & 6 & 56.69 & 1.55 & 0.18 & 1 & 0.893 & 1.050 \\
\hline 4.2 & 1.6 & 1.48 & 0.96 & 0.22 & 8 & 58.78 & 1.33 & 0.15 & 0 & 0.462 & 0.161 \\
\hline 6.2 & 1.6 & 1.63 & 0.96 & 0.22 & 5 & 52.85 & 1.17 & 0.15 & 1 & 0.821 & 0.675 \\
\hline 8.2 & 1.6 & 1.72 & 0.93 & 0.22 & 20 & 73.05 & 1.05 & 0.15 & 0 & -0.182 & 0.096 \\
\hline 10.2 & 1.6 & 1.78 & 0.9 & 0.22 & 14 & 64.59 & 0.95 & 0.15 & 0 & 0.481 & 0.096 \\
\hline 2.2 & 0.5 & 1.68 & 0.98 & 0.12 & 2 & 50.53 & 1.74 & 0.09 & 0 & 0.212 & 0.104 \\
\hline 3.7 & 0.5 & 1.82 & 0.98 & 0.12 & 11 & 63.92 & 1.50 & 0.09 & 0 & -0.211 & 0.207 \\
\hline 6.2 & 0.5 & 1.92 & 0.96 & 0.12 & 11 & 62.77 & 1.25 & 0.09 & 0 & -0.187 & 0.214 \\
\hline 8.2 & 0.5 & 1.96 & 0.93 & 0.12 & 5 & 52.36 & 1.11 & 0.09 & 0 & -0.151 & 0.098 \\
\hline 9.2 & 0.5 & 1.97 & 0.91 & 0.12 & 18 & 70.69 & 1.05 & 0.09 & 0 & -0.189 & 0.098 \\
\hline 6.0 & 1.0 & 1.77 & 0.96 & 0.22 & 8 & 58.20 & 1.23 & 0.20 & 1 & 1.028 & 0.595 \\
\hline 6.0 & 1.0 & 1.77 & 0.96 & 0.22 & 11 & 62.63 & 1.23 & 0.29 & 1 & 1.148 & 0.526 \\
\hline 6.0 & 1.0 & 1.77 & 0.96 & 0.12 & 10 & 61.2 & 1.23 & 0.21 & 1 & 0.985 & 0.658 \\
\hline 6.0 & 1.0 & 1.77 & 0.96 & 0.12 & 8 & 58.2 & 1.23 & 0.21 & 1 & 0.738 & 1.060 \\
\hline 6.0 & 1.0 & 1.77 & 0.96 & 0.12 & 12 & 64.03 & 1.23 & 0.16 & 1 & 0.431 & 0.100 \\
\hline 6.0 & 1.0 & 1.77 & 0.96 & 0.12 & 13 & 64.09 & 1.23 & 0.23 & 1 & 0.931 & 0.817 \\
\hline 6.0 & 1.0 & 1.77 & 0.96 & 0.12 & 14 & 66.73 & 1.23 & 0.21 & 1 & 0.726 & 0.119 \\
\hline 6.0 & 1.0 & 1.77 & 0.96 & 0.22 & 13 & 65.39 & 1.23 & 0.16 & 1 & 0.249 & 0.545 \\
\hline 6.0 & 1.0 & 1.77 & 0.96 & 0.22 & 16 & 69.30 & 1.23 & 0.25 & 1 & 0.577 & 0.602 \\
\hline 6.0 & 1.0 & 1.77 & 0.96 & 0.22 & 12 & 64.03 & 1.23 & 0.33 & 1 & 1.145 & 1.07 \\
\hline 6.0 & 1.0 & 1.77 & 0.96 & 0.22 & 20 & 74.15 & 1.23 & 0.35 & 1 & 0.111 & 0.109 \\
\hline 6.0 & 1.0 & 1.77 & 0.96 & 0.22 & 13 & 65.39 & 1.23 & 0.22 & 1 & 0.839 & 0.537 \\
\hline 6.0 & 1.0 & 1.77 & 0.96 & 0.12 & 14 & 66.73 & 1.23 & 0.12 & 1 & -0.067 & 0.208 \\
\hline 6.0 & 1.0 & 1.77 & 0.96 & 0.12 & 14 & 66.73 & 1.23 & 0.09 & 1 & -0.167 & 0.208 \\
\hline
\end{tabular}


Table 5. the error of prediction with reference model $(\mathrm{Wu}$, 1995)

\begin{tabular}{|c|c|c|c|c|}
\hline & & $\begin{array}{l}\text { Actual behavior: } \\
\text { Liquefaction } \\
\text { Model prediction: } \\
\text { No liquefaction } \\
(\%)\end{array}$ & $\begin{array}{l}\text { Actual behavior: } \\
\text { No liquefaction } \\
\text { Model prediction: } \\
\text { Liquefaction } \\
(\%)\end{array}$ & Average (\%) \\
\hline \multirow{2}{*}{$\begin{array}{c}\text { Reference } \\
\text { neural network }\end{array}$} & $\begin{array}{l}\text { Training set } \\
\left(55^{* *} 45 *\right)\end{array}$ & 5.45 & 6.67 & 6 \\
\hline & $\begin{array}{l}\text { Testing set } \\
(28 * * 21 *)\end{array}$ & 21.43 & 14.29 & 18.37 \\
\hline \multirow{2}{*}{$\begin{array}{l}\text { Proposed } \\
\text { Fuzz-ART }\end{array}$} & $\begin{array}{l}\text { Training set } \\
\left(55^{* *} 45^{*}\right)\end{array}$ & 1.82 & 0 & 1 \\
\hline & $\begin{array}{l}\text { Testing set } \\
\left(28^{* *} 21^{*}\right)\end{array}$ & 21.43 & 0 & 12.24 \\
\hline
\end{tabular}

(actual soil behavior: no liquefaction)* (actual soil behavior: liquefaction)**

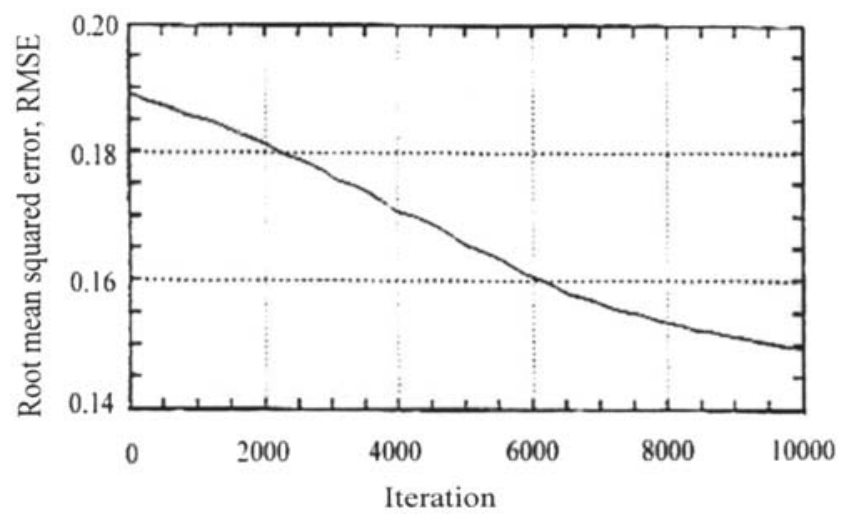

Fig. 5. Learning curve of reference neural network (Wu, 1995).

model. It shows that besides higher successive rate, the proposed model converges much quicker than conventional Wu's 3 layer neural network model.

\section{LIQUEFACTION EVALUATION DURING CHI-CHI EARTHQUAKE IN YUAN-LIN AREA}

After 1999 Chi-Chi earthquake, National Center for Research on Earthquake Engineering (NCREE) supported several projects for liquefaction study in areas of Da Chun, Yuan Lin, Sheh Tou. Many SPT and CPT were conducted for evaluation of liquefaction potential in those areas (Moh and Associated, 1999), several empirical methods are available in the literature for evaluation of liquefaction induced by Chi-Chi earthquake. Idriss presented charts (Fig. 7) of observations regarding liquefaction during 1999 Chi-Chi earthquake (Idriss 2000). As shown in Fig. 7, there are 79 data points in Idriss's collection. Among them 4 data points of liquefaction potential proposed falls in nonliquefaction zone with successive prediction rate of

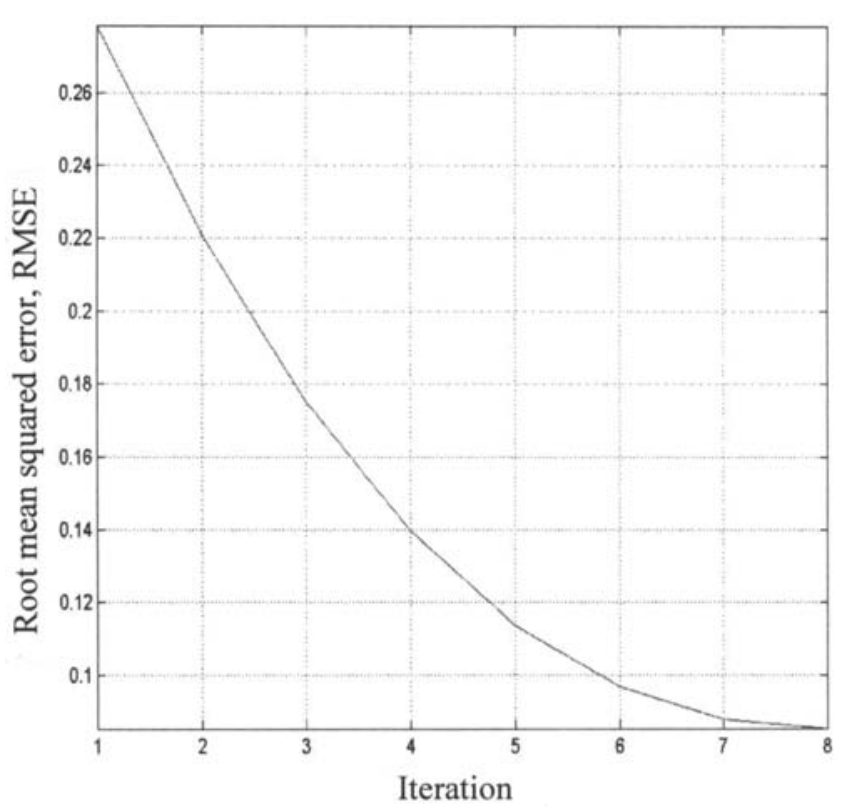

Fig. 6. Learning curve of Fuzz-ART.

$90.7 \%$ and 10 data points of non-liquefaction falls in liquefaction zone, with successive predication rate of $71.4 \%$. The data used in the present study are also come from Moh and Associated's results of subsurface exploration; however, they are different from Idriss'. A total of 42 boring data in Yuan-Lin area is considered due to limitation of subsurface explorations. The results of predictions using the Fuzz-ART model is shown in Table 6 , there are 6 errors in training data and 2 errors in testing data $(72.7 \%$ success rate in training and $90 \%$ success rate in testing). Due to insufficient data, the success rate in predicating liquefaction in Yuan-Lin area by using proposed Fuzz-ART neural network method is not so satisfied as compared with case studies presented above, however, the results will be improved as data provided increases.

\section{CONCLUSION}

Fuzz-ART neural network has been used to model the complex relationship between the seismic and soil parameters, and the liquefaction potential, using actual field records. Comparisons from case studies indicate that Fuzzy-ART neural network model is more reliable than the methods of conventional three layer neural networks. The results of proposed method will improve as more input data are provided in Yuan-Lin area.

\section{REFERENCES}

1. Qshaki, Y., "Effects of Sand Compaction on Liquefac- 
Table 6a. Chi-Chi earthquake field behavior data for training set

(1: liquefaction $\quad 0$ : no liquefaction)

\begin{tabular}{rrrrrrrrrr}
\hline \multicolumn{1}{c}{ Depth } & \multicolumn{1}{c}{$\sigma_{v}$} & $\mathrm{~N}_{1}$ & $\tau_{a v} / \sigma_{v}^{\prime}$ & $\gamma_{d}$ & $\tau_{a v}$ & $\mathrm{FC}$ & $D_{5}$ & $\begin{array}{c}\text { Field } \\
\text { behavior }\end{array}$ & $\begin{array}{c}\text { Fuzz-ART } \\
\text { model* }\end{array}$ \\
\hline 13.275 & 14.89 & 7.8 & 0.094 & 0.82 & 3.56 & 17.5 & 0.15 & 0 & 0.009 \\
11.775 & 11.42 & 11.1 & 0.129 & 0.85 & 3.21 & 97.8 & 0.01 & 1 & 0.009 \\
14.775 & 15.01 & 6.2 & 0.075 & 0.78 & 3.79 & 44.0 & 0.07 & 1 & 0.009 \\
5.775 & 5.81 & 3.9 & 0.046 & 0.96 & 1.81 & 47.6 & 0.07 & 0 & -0.142 \\
10.275 & 11.92 & 5.0 & 0.060 & 0.88 & 2.96 & 32.0 & 0.09 & 1 & 0.009 \\
19.275 & 22.79 & 6.1 & 0.073 & 0.68 & 4.60 & 28.8 & 0.18 & 1 & 0.200 \\
6.775 & 7.81 & 7.9 & 0.095 & 0.95 & 2.15 & 15.4 & 0.35 & 1 & 0.200 \\
14.775 & 17.30 & 7.0 & 0.084 & 0.78 & 3.67 & 23.2 & 0.10 & 0 & 0.009 \\
14.775 & 16.66 & 10.1 & 0.119 & 0.78 & 3.88 & 30.7 & 0.10 & 0 & 0.009 \\
5.775 & 6.53 & 6.2 & 0.074 & 0.96 & 1.76 & 20.3 & 0.46 & 0 & -0.151 \\
12.775 & 15.72 & 7.2 & 0.086 & 0.83 & 3.50 & 25.6 & 0.12 & 0 & 0.009 \\
18.275 & 20.91 & 7.2 & 0.086 & 0.70 & 4.37 & 7.8 & 0.16 & 1 & 0.193 \\
7.275 & 7.90 & 4.5 & 0.054 & 0.94 & 2.17 & 45.4 & 0.65 & 0 & -0.151 \\
4.275 & 6.09 & 7.6 & 0.091 & 0.98 & 1.51 & 33.5 & 0.13 & 1 & 0.193 \\
11.775 & 11.71 & 16.5 & 0.183 & 0.85 & 3.24 & 11.5 & 0.62 & 1 & 0.203 \\
14.775 & 16.04 & 5.9 & 0.072 & 0.78 & 3.80 & 33.4 & 0.09 & 1 & 0.009 \\
8.775 & 7.45 & 7.0 & 0.083 & 0.91 & 2.41 & 96.6 & 0.09 & 0 & -0.096 \\
8.775 & 9.48 & 5.1 & 0.062 & 0.91 & 2.59 & 32.7 & 0.16 & 0 & 0.009 \\
7.275 & 7.46 & 5.8 & 0.070 & 0.94 & 2.12 & 28.5 & 0.87 & 1 & 0.009 \\
11.775 & 12.19 & 10.7 & 0.125 & 0.85 & 3.29 & 17.5 & 0.22 & 1 & 0.009 \\
16.275 & 17.65 & 7.3 & 0.087 & 0.75 & 3.99 & 30.1 & 0.18 & 1 & 0.193 \\
19.275 & 25.25 & 6.0 & 0.072 & 0.68 & 4.28 & 48.6 & 0.08 & 0 & 0.009 \\
\hline
\end{tabular}

The shadow determinates the error of prediction $*$ not normalized between 0 and 1

Table 6b. Chi-Chi earthquake field behavior data for testing set

(1: liquefaction $\quad 0$ : no liquefaction)

\begin{tabular}{rrrrrrrrrr}
\hline Depth & \multicolumn{1}{c}{$\sigma_{v}$} & $\mathrm{~N}_{1}$ & $\tau_{a v} / \sigma_{v}^{\prime}$ & $\gamma_{d}$ & $\tau_{a v}$ & $\mathrm{FC}$ & $D_{5}$ & $\begin{array}{c}\text { Field } \\
\text { behavior }\end{array}$ & $\begin{array}{c}\text { Fuzz-ART } \\
\text { model* }\end{array}$ \\
\hline 7.275 & 9.49 & 7.2 & 0.086 & 0.94 & 2.11 & 82.1 & 0.04 & 0 & 0.0088 \\
13.275 & 21.12 & 6.5 & 0.079 & 0.82 & 3.77 & 41.0 & 0.16 & 0 & -0.1490 \\
16.225 & 16.04 & 9.7 & 0.114 & 0.75 & 4.00 & 12.4 & 0.32 & 1 & 0.1997 \\
4.765 & 4.76 & 7.0 & 0.084 & 0.97 & 1.44 & 49.7 & 0.08 & 0 & -0.1448 \\
14.775 & 15.61 & 8.3 & 0.099 & 0.78 & 3.81 & 19.6 & 2.63 & 1 & 0.0075 \\
19.275 & 19.60 & 5.7 & 0.069 & 0.68 & 4.28 & 67.5 & 0.05 & 0 & 0.0082 \\
5.775 & 5.47 & 8.0 & 0.095 & 0.96 & 1.69 & 9.9 & 0.28 & 0 & 0.0006 \\
8.775 & 9.25 & 6.8 & 0.081 & 0.91 & 2.55 & 36.8 & 0.12 & 0 & -0.1831 \\
11.775 & 11.83 & 10.0 & 0.117 & 0.85 & 3.31 & 11.3 & 0.38 & 0 & 0.0082 \\
8.775 & 9.81 & 3.5 & 0.041 & 0.91 & 2.60 & 30.3 & 0.11 & 0 & 0.0007 \\
5.775 & 7.38 & 3.5 & 0.041 & 0.96 & 1.72 & 33.7 & 0.11 & 0 & -0.0929 \\
10.275 & 11.12 & 10.4 & 0.121 & 0.88 & 2.91 & 28.4 & 0.12 & 0 & 0.0089 \\
10.275 & 11.12 & 10.4 & 0.121 & 0.88 & 2.91 & 14.8 & 0.13 & 0 & -0.1421 \\
17.775 & 18.81 & 10.5 & 0.123 & 0.72 & 4.17 & 11.9 & 0.17 & 0 & -0.1473 \\
47.775 & 57.67 & 1.8 & 0.018 & 1.43 & 24.65 & 27.0 & 0.13 & 0 & 0.0089 \\
11.775 & 14.35 & 8.0 & 0.096 & 0.85 & 3.31 & 23.7 & 0.12 & 0 & -0.0956 \\
13.275 & 12.25 & 8.9 & 0.105 & 0.82 & 3.43 & 30.5 & 0.09 & 1 & 0.0089 \\
8.775 & 10.95 & 3.8 & 0.045 & 0.91 & 2.71 & 45.4 & 0.12 & 0 & -0.1375 \\
14.775 & 15.75 & 8.3 & 0.099 & 0.78 & 3.93 & 10.2 & 0.17 & 0 & 0.0089 \\
4.275 & 6.74 & 2.4 & 0.027 & 0.98 & 1.40 & 16.1 & 0.17 & 1 & 0.1997 \\
\hline
\end{tabular}

The shadow determinates the error of prediction *not normalized between 0 and 1 


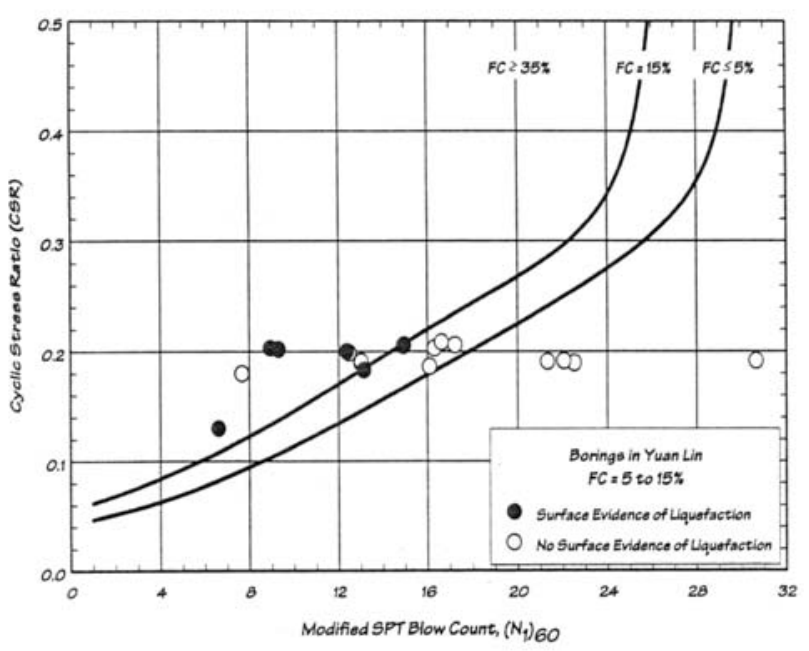

(a)

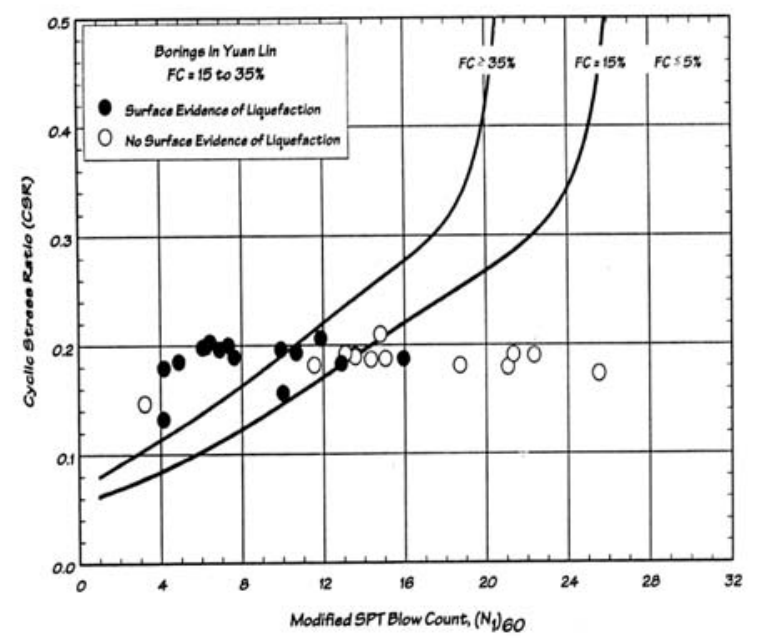

(b)

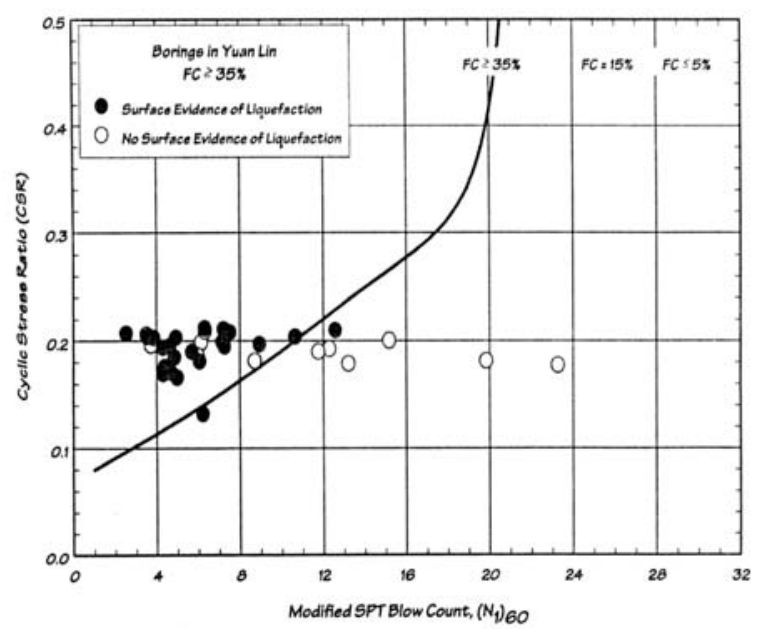

(c) tion During the Jokackioki Earthquake," Soils and Foundation, Vol. 32, No. 2, pp. 112-128 (1970).

2. Yomamuro, J.A. and Lade, P.V., "Instability of Granular Materials at High Pressures," Soils and Foundations, Vol. 37, No. 1, pp. 41-51 (1997).

3. NCREE, NAPHM, and Taiwan Geotechnical Society, Reconnaissance Report of the Geotechnical Hazard Caused by Chi-Chi Earthquake, Taipei, Taiwan (1999) (in Chinese).

4. Seed, H.B. and Idriss, I.M., "Simplified Procedure for Evaluating Soil Liquefaction Potential," ASCE Journal of SM and Found., 97(SM9), pp. 1249-1273 (1974).

5. Tokimatsu, K. and Yoshimi, Y., "Empirical Correlation of Soil Liquefaction Based on SPT-N Value and Fines Content," Soils and Foundation, Vol. 23, No. 4, pp. 5674 (1983).

6. Robertson, P.K., Woeller, D.J. and Finn, W.D.L., "Seismic Cone Penetration Test for Evaluating Liquefaction Potential under Cyclic Loading," Canadian Geotechnical Journal, Vol. 29, pp. 686-695 (1992).

7. Goh, A.T.C., "Seismic Liquefaction Potential Assessed by Neural Network," ASCE Journal of Geotechnical Engineering, Vol. 120, No. 9, pp. 1467-1480 (1994).

8. Juang, C.H. and Chen, C.J. "A Rotational Method for Development of Limit State for Liquefaction Evaluation Based on Shear Wave Velocity Measurements," Int. J. Numer. Anal. Meth. Geomech, Vol. 24, pp. 1-27 (2000).

9. Wu, J.Y., "Neural Network Evaluation of Liquefaction Potential," Master Thesis, National Taiwan University, Taipei, (1995) (in Chinese).

10. Carpenter, G.A., Grossberg, S. and Rosen, D.B., "Fuzzy ART: fast stable learning and categorization of analog patterns by an adaptive resonance system," Neural Networks, Vol. 4, pp. 759-771 (1991).

11. Lin, C.T., Lin, C.J. and Lee, C.S.G., "Fuzzy Adaptive Learning Control Network with On-Line Neural Learning," Fuzzy Sets and Systems, Vol. 71, pp. 25-45 (1995).

12. Lin, C.J. and Lin, C.T., "An ART-Based Fuzzy Adaptive Learning Control Network," IEEE Transaction on Fuzzy Systems, Vol. 5, No. 4, pp. 477-496 (1997).

13. MAA, Soil Liquefaction Evaluation and Mitigation Study, Phase I (Yuen-Lin, Da-Chun and Sheh-Tou), Final Report to National Science Council, April 2000 (2000) (in Chinese).

14. Idriss, I.M., "A Preliminary Observations Regarding Liquefaction During the 1999 Chi-Chi Earthquake," Notes for Presentation, National Taiwan University of Science and Technology (2000).

Fig. 7. Relationship between cyclic stress ratio and modified blow count (a) $\mathrm{FC}=5-15 \%$ (b) $\mathrm{FC}=15-35 \%$ (c) FC $35 \%$ (Idriss, 2000). 


\title{
模糊自適應共振類神經網路預估集 \\ 集大地震中員林地區之液化潛能
}

\author{
陳 俶 季
}

國立臺灣海洋大學河海工程學系

胡第豐張延任蔡宜峰

國立臺灣海洋大學河海工程學系研究所

\section{摘 要}

本文以自適應共振理論(ART)爲構思基礎, 結 合模糊系統理論, 提出模糊推理類神經網路“FuzzART”來評估集集大地震中員林地區的液化潛能。本 研究模式以倒傳彪演算法作爲參數學習機制及以模糊 自適應共振理論(fuzzy ART)作爲架構學習機制。經 由案例分析結果顯示, “Fuzz-ART”預估液化潛能可 得到較傅統類神經網路更爲準確有效之評估結果。若 能收集更多關於集集地震的資料, 此架構對員林地區 之液化潛能將能得到更爲良好之評估效果。 